\title{
北京东灵山区两种生境条件下辽东栎 幼苗补充与建立的比较
}

高贤明 杜晓军 王中磊

(中国科学院植物研究所植被数量生态学重点实验室 北京 100093)

摘 要 北京东灵山区的人工油松 (Pinus tabulaeformis) 林中常常混生有萌生的辽东栋 ( Quercus wutaishanica) 种群。 局部地带辽东栎与油松形成混交林,在山脊及山坡上部一些特殊生境辽东栎甚至取代油松林成为优势种。为了探 讨北京东灵山区辽东栎林的天然更新机制及辽东栎幼苗在人工油松林中的天然更新，在油松人工林的林下和林缘 两种生境条件下将辽东栎种子播种于 $2 \mathrm{~cm}$ 深的土中，对辽东栎幼苗的补充和建立进行了对照试验。结果显示，在 辽东柇结实丰年, 两种生境条件下播种后辽东栎种子丢失差异并不显著, 而辽东栎幼苗补充和建立方面的差异极 显著。由于森林动物特别是啮齿类动物的活动对辽东栎幼苗建立的影响具有二重性 消耗种子与促进萌发, 本试 验特别关注辽东栋幼苗子叶丢失现象及其丢失后果。由于辽东栎种子萌根较早, 在幼苗出土前其粗大的主根长达 $10 \sim 20 \mathrm{~cm}$, 并已初步形成根系, 表明已有相当比例的营养物质从子叶转移到根部。因此当幼苗出土后如果仅仅只 是子叶的丢失对辽东栎幼苗的成活和生长均无明显的影响。通过研究发现辽东栎幼苗期子叶丢失有 3 种形式:1) 仅丢失子叶 幼苗其它部位未受伤害, 多发生在林缘生境中, 占发芽坚果的 $29.69 \%$, 2) 整个幼苗连根被拖出地面， 多发生在林下生境，占 $62.43 \%$ 3) 在子叶与根颈的连接处主干被咬断，林缘为 $50.88 \%$, 显著高于林下的 $8.41 \%$ 。 但只有后两种形式才导致幼苗死亡。导致这些现象的原因是由于林缘土壤腐殖质含量低, 比较干燥, 土质较坚实, 以及幼苗出土前坚果主根生长得较长等缘故, 当幼苗遭受动物捕食时不会轻易地被拖出地面, 拖走的往往仅仅是 子叶。至生长季节结束 林缘样方辽东栎幼苗的成活率接近 $20 \%$, 茎干平均高度 $7.94 \mathrm{~cm}$ 芽数 11.24 株 $^{-1}$, 叶片平 均干重为 $81.14 \mathrm{mg}$ 株 ${ }^{-1}$, 且几乎全部由丢失子叶的幼苗所构成; 而林下样方辽东栋幼苗的成活率不足 $2 \%$, 茎干平 均高度 $4.74 \mathrm{~cm}$ 芽数 7.52 株-1 叶片平均干重仅为 $42.27 \mathrm{mg}$ 株 ${ }^{-1}$, 以未丢失子叶的幼苗为主。试验结果表明 林 缘及类似林缘的环境条件更有利于辽东柇的实生更新。

关键词 辽东柇坚果 子叶丢失 生境 幼苗补充与建立 东灵山

\section{COMPARISON OF SEEDLING RECRUITMENT AND ESTABLISHMENT OF QUERCUS WUTAISHANICA IN TWO HABITATS IN DONGLING MOUNTAINOUS AREA , BEIJING}

\author{
GAO Xian-Ming DU Xiao-Jun and WANG Zhong-Lei \\ ( Laboratory of Quantitative Vegetation Ecology, Institute of Botany , the Chinese Academy of Sciences , Beijing 100093 , China)
}

\begin{abstract}
In Beijing Dongling Mountainous area , the coppiced populations of Liaodong oak ( Quercus wutaishanica Mayr) usually appear as an associated tree species of the Chinese pine (Pinus tabulaeformis Carr. ) artificial forest. In some places the artificial pine forest is replaced by mixed crops of Chinese pine and Liaodong oak, or the oak takes the place of the pine as the dominant species in some specific habitats along the ridges and the upper slopes of hills. In order to compare and understand the recruitment and establishment of Liaodong oak seedlings under Chinese pine artificial forest as well as the natural regeneration mechanism of the oak forest, an artificial forest of Chinese pine with Liaodong oak as an associated species in Beijing Dongling Mountainous area was selected, and comparative experiments on the recruitment and establishment of seedlings were conducted by sowing the acorns of Liaodong oak about $2 \mathrm{~cm}$ below the floor surface in two habitats , under the canopies and along the boundaries of the artificial forest. The results showed that in the mast year of the oak , though the difference of loss of the acorns planted in these two habitats was not significant, yet there were highly significant differences in the recruitment and establishment of seedlings. Since activities of small forest animals, rodents in particular, have both positive and negative effects on oak forest regeneration, through acorn consumption and germination facilitation, close attention was paid to the phenomenon of cotyledon loss of
\end{abstract}


acorns and the consequences of the loss. As the acorns germinated as soon as they fell from the mother tree in autumn , before the seedlings sprouted their caudices , the taproots would have grown 10-20 cm long and formed primary root systems, implying that certain amount of the nutrition stored in the cotyledons had been transported to the roots. From the field experiments it was found that the acorn cotyledons were consumed by the predator in three ways during the early seedling stage : 1) the seedlings lost their cotyledons without harming any other parts, which often occurred along the forest boundaries, accounting for $29.69 \%$ of the germinated acorns ;2) the whole seedlings were drawn out of the soil, which almost always happened under the forest canopies , accounting for $62.43 \% ; 3$ ) the stems of seedlings were broken at the bases where the cotyledons are connected, occurring $50.88 \%$ along the boundaries, more frequently than under the forest canopies where only $8.41 \%$ of germinated acorns occurred. Only the latter two ways caused the seedlings to die, while cotyledon loss after seedling emergence from the soil had little negative effects on the growth and survival of the seedlings. These phenomena might result from different soils between these two habitats ; soil along the boundaries has little humus and is much harder and drier than that of the forest floor under the canopy. Also, the taproots were much longer along the boundaries. So when rodents predate the seedlings along the boundaries , it would be easier to split the cotyledons from the seedlings instead of pulling out the whole root from the soil. At the end of the first living period , the survival rate of the seedlings along the boundaries was close to $20 \%$, the average height of the stems reached $7.94 \mathrm{~cm}$, average buds were 11.24 per seedling, and the average dry weight of leaves was $81.14 \mathrm{mg}$ per seedling. Moreover, almost all of the surviving seedlings along the boundaries lost their cotyledons. The survival rate under forest canopies was less than $2 \%$, the average height of stems reached $4.74 \mathrm{~cm}$, average buds were 7.52 per seedling, and the dry weight of leaves was only 42.27 mg per seedling, but the surviving seedlings under canopy did not lose their cotyledons. The above results showed that the habitats along the forest boundaries and similar areas would be more beneficial to the recruitment and establishment of oak seedlings.

Key words Quercus wutaishanica, Acorn , Cotyledon loss, Seedling, Habitat, Recruitment and establishment , Dongling Mountainous area

林下更新苗匮乏是许多落叶栎林的共同特征 (Cho \& Boermer，1991 ;王巍，2000)。由于栎类坚果 (通常被称之为种子) 是昆虫、乌类、啮齿类等动物以 及真菌等微生物的重要食物源, 因此从坚果成长、成 熟、脱落、萌发到幼苗期间一直遭受着巨大的捕食压 力从而导致栎林实生更新困难。早在 20 世纪初 期,Watt(1919)就注意到，在结实大年的秋季栋林下 的地表会被成熟后自然落下的大量栎树坚果所覆 盖, 并能够在第二年顺利地从枯枝落叶或草丛中萌 发出成千上万的幼苗, 但即使是这样 林下多年生栎 树幼苗幼树仍然十分贫乏。在强大的捕食压力下， 栎树多采取以萌葟更新的对策(Ducrey \& Toth ,1992 ; Giovannini et al . 1992;Bacilieri et al . 1993;Espelta et $a l$, 1995; 王巍等, 2000 ;孙书存等, 2001; 高贤明等， 2001)。然而，作为维持种群的遗传多样性和生产力 及林木健康的有效途径, 适应捕食压力, 完成实生更 新是栎林维持不可缺少的重要环节。

北京东灵山区的油松 (Pinus tabulaeformis) 人工 林是 20 世纪 70 年代前后营造的, 造林地主要是辽 东栎林或以辽东栋为优势种的落叶阔叶混交林砍伐 迹地。因此目前油松人工林中通常混生有辽东栎

( Quercus wutaishanica, 常用异名: $Q$. liaotungensis, 见 :中国植物志 22 卷) 萌生个体, 在部分地段形成油
松辽东栎混交林, 甚至在比较干旱瘦薄的山脊和山 坡上部形成小面积以辽东柇为优势种的森林类型。 目前东灵山区油松人工林中除了辽东栋萌生个体 外, 尚有一定数量的辽东栎实生苗, 尤其在林缘多见 (孙书存, 1998; 王巍等,2000 孙书存等 ,2001)。

在栎林天然更新研究中，有关捕食动物对栎类 种子的消耗、搬运、埋藏、传播等方面报道很多(如： Shaw , 1968a ; 1968b ; Crawley \& Long , 1994 ; Chambers \& Mackahon , 1994 ; Kollmann \& Schill , 1996) ,而关于 栎树种子萌芽前子叶营养向根部的转移及出土时的 子叶丢失对栎树幼苗建立的影响关注较少。作为唯 一的营养储备器官, 无胚乳种子的子叶对幼苗早期 生长具有十分重要的意义。栎属 ( Quercus) 植物的 种子无胚乳, 子叶肥大, 富含淀粉等营养物质, 是啮 齿类、鸟类等森林动物的重要食物来源 ( Jones， 1959)。森林动物对栎类坚果的捕食消耗不仅发生 在坚果下落的种子雨阶段, 而且还发生在栎树幼苗 补充的早期阶段，即对栎树幼苗期种子 (坚果) 子叶 的捕食消耗 (Bossema，1979)，因此栎树幼苗期子叶 丢失现象比较普遍。但是, 出土后子叶的丢失是否 对栎树幼苗的成活与生长产生不良影响目前还存在 着较大争议。Ovington 和 MacRae(1960) 及 Brookes 等 (1980)学者认为, 子叶丢失会造成係树幼苗成活率 
与生长速率的大幅度下降, 但也有试验结果显示, 这 种影响微不足道 (Sonesson ,1994) ;Brookes(1976 引自 Sonesson, 1994) 认为, 在贫痊的土壤上栎树幼苗的第 一年生长会更多地依赖于子叶中营养的储备, 而 Jarvis(1963)却发现，栎树幼苗生长与土壤营养状况 并无相关关系。尽管动物捕食消耗了大量的栎树种 子，但动物捕食过程中的搬运、埋藏等活动对栎树的 更新和散布也非常重要 (Ovington \& MacRae,1960； Sonesson , 1994 ; Kollmann \& Schill, 1996 ; 王巍等， 2000 孙书存等,2001;Zhang \& Wang,2001), ,因此有 人曾推测 栎树种子与作为捕食者及传播者的动物 之间, 可能存在着一定程度的补偿平衡 (Trade-off) 关 系 即柇树对这些动物传播种子的依赖和对它们捕 食压力的适应 (Price \& Jendins, 1986 ; Kollmann \& Schill , 1996)。

本文在油松人工林下和林缘两种生境条件下进 行播种试验, 对辽东栎坚果及幼苗补充进行野外比 较研究, 以了解这两种生境条件下辽东栋 1) 种子秋 季萌根及幼苗出土前主根生长状况,2) 幼苗早期子 叶丢失情况及其对幼苗补充与建立的影响 ,3) 幼苗 生长速率, 并通过对上述问题的回答进一步探讨 4) 辽东柇幼苗适应动物捕食压力的对策。

\section{1 研究地点及油松人工林群落特征}

野外工作在位于北京西部东灵山区、距北京市 区 $114 \mathrm{~km}$ 的中国科学院北京森林生态系统定位研 究站 $\left(115^{\circ} 26^{\prime} \mathrm{E}, 40^{\circ} 00^{\prime} \mathrm{N}\right)$ 进行。东灵山为太行山系 小五台山脉的东延余脉，属暖温带大陆性季风气候。 据气象观测资料统计，东灵山地区年平均气温 4.8 ${ }^{\circ} \mathrm{C}$, 最热月 7 月, 平均气温 $18.3{ }^{\circ} \mathrm{C}$, 最冷月 1 月, 平 均气温 $-10.1{ }^{\circ} \mathrm{C}$; 年降水量 $611.9 \mathrm{~mm}, 6.7,8$ 三个月 降水比较集中,占全年降水量的 $78 \%$ (茅世森等, 1997)。

试验样地选择油松 (Pinus tabulaeformis))生长较 旺盛的山坡中下部, 坡向东偏南 $15^{\circ}$ 坡度 $25^{\circ} \sim 35^{\circ}$; 林缘地带地势平坦。群落以油松占绝对优势, 沿沟 谷山麓有较多的核桃楸 (Juglans mandshurica) 和少量 的青杨 (Populus cathayana) 分布。油松人工林乔木 层盖度约 $90 \%$, 其中油松和辽东栎的高度均约在 12 $\mathrm{m}$ 上下, 辽东栎平均胸径 $14.3 \mathrm{~cm}$, 不同地段盖度变 化幅度在 5\% 60\%之间，但一般不超过 10\% 核桃 楸稍高，约 $14 \mathrm{~m}$; 青杨高度则可达 $18 \mathrm{~m}$ 。灌木层盖 度 $10 \%$ 左右, 组成种类很少, 较常见的是胡枝子 (Lespedeza bicolor)、柔毛绣线菊 ( Spiraea pubescens)、雀
儿舌头( Andrachne chinensis) ,偶见大叶白蜡( Fraxinus rhynchophylla)、山桃 ( Prunus davidiana)、照山白 (Rhododendron micranthum) 等。草本层盖度 10\% $25 \%$, 最常见的是野青茅 (Calamagrostis arundinacea )、小红菊 (Dendranthema chanetii)、大油芒 (Spodiopogon sibiricus), 其次还有石竹 (Dianthus chinensis)、堇菜(Viola spp.) 等。

在林缘，乔木层以青杨为主要伴生种，其次还有 少量的核桃楸和辽东栎，层总盖度约 $80 \%$,但辽东 栎数量很少, 树高可达 $15 \mathrm{~m}$, 平均胸径 $9.6 \mathrm{~cm}$, 盖度 只有 $1 \% \sim 5 \%$ 。由于光照条件较好, 草本层比较发 达。灌木层主要是胡枝子, 柔毛绣线菊较少, 层盖度 变动范围在 $20 \% \sim 45 \%$ 左右。草本层以大油芒为 主, 蓝萼香茶菜 ( Rabdosia japonica var. glaucocalyx)、 水棘针 (Amethystea caerulea)、蒿 (Artemisia spp.)、委 陵菜 (Potentilla spp.)、堇菜等也较常见，层盖度约 $50 \%$ 。林缘枯枝落叶层厚度通常不足 $1 \mathrm{~cm}$, 以阔叶 树的枯枝落叶和枯草为主, 土壤因腐殖质含量低而 色浅; 而油松人工林下则达 $2 \sim 3 \mathrm{~cm}$, 主要为油松的 针叶, 土壤富含腐殖质而呈深褐色。由于本试验位 于山体的偏南坡 林缘直射光强度远远高于林下。

\section{2 研究方法}

\section{1 辽东栎坚果的收集与选择}

2000 年为辽东柇结实大年, 种子雨于 8 月份开 始 8 月底进入高峰期, 至 9 月上、中旬辽东栎林地 地面几乎为辽东柇坚果所覆盖。在辽东栎坚果雨高 峰期收集辽东栎成熟坚果 (橡实 种子) ,挑选大小相

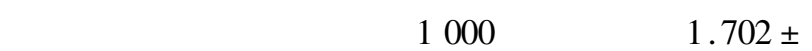
$0.144) \mathrm{cm}$,直径 $(1.508 \pm 0.136) \mathrm{cm}$,鲜重 $(2.370 \pm$ $0.595) \mathrm{g}, n=120$,千粒重 $2401 \mathrm{~g}$ ), 用于播种试验。

\section{2 样方设置与播种}

在油松人工林林下和林缘分别设置 $20 \mathrm{~cm} \times 20$ $\mathrm{cm}$ 样方各 20 个, 分别将 20 粒辽东栎坚果播于土 中 覆土 $2 \mathrm{~cm}$, 然后再覆以与周围同等厚度的枯枝 落叶。在林下和林缘样方附近各设置 8 个装满与样 方相同土壤、口径 $6 \mathrm{~cm}$ 、高 $20 \mathrm{~cm}$ 、底部有孔(孔直径 $1.5 \mathrm{~cm}$ ) 的透明容器 将 8 粒辽东栎坚果按上述相同 的播种深度播于其中, 并使坚果顶端 (胚根伸出处) 朝向容器的内壁, 以利于观测坚果发芽情况和发芽 后主根生长速度, 然后埋入土中并使容器口与地面 在同一水平上或略低;观察时从土中挖出容器, 测量 后随即埋回原处。由于容器底部有较大的孔, 在东 灵山这样雨季集中在 $6 、 7 、 8$ 三个月 (茅世森等, 
1997)的山区 秋冬季节埋入土中则容器内外的土壤 含水量、温度等条件能够基本保持一致。容器中辽 东栎种子胚根萌发长度的测量由于时间较短, 因此 可以认为容器的使用及容器中幼根的测量, 均不会 对容器中土壤理化性质和幼根的生长产生明显的影 响。

\section{3 调查}

辽东栎坚果留存的调查始于 2000 年 9 月 9 日， 除少数雨天外, 调查工作于每天上午进行, 一直延续 到 2000 年 11 月 4 日。调查内容主要是容器内辽东 栎坚果发芽、主根生长情况 根据样方地面啮齿类扒 土现状检查确定各样方坚果丢失数量。辽东栋幼苗 的发芽出土前后啮齿类捕食情况和幼苗高生长的观 测始于 2001 年 5 月 16 日，每周 1 次。所有观测直至 2001 年 9 月 7 日结束, 观测内容包括坚果留存数、已 发芽坚果数、根据幼苗根部土壤是否有动物扒过的 痕迹检查子叶丢失情况、幼苗高度、死亡数及致死原 因, 以及容器内坚果冬季前胚根的萌发、生长情况 等, 并在第一次调查幼苗出土情况时挖容器中的幼 苗，以观察根系发育状况并测定其主根的长度。播 种 4 周后由于大部分坚果已经萌发生根, 为了避免 对萌发过程产生不良影响, 萌发情况的刨土调查终 止。有关幼苗比率的数据仅以出土幼苗计。对每一 株出土后的幼苗每 2 天测量一次苗高, 直至出土后 约 2 周 (高生长结束)。幼苗出土时挖出容器, 清理 出容器中的辽东栎种子 (包括根、子叶等), 用水洗去 泥土晾干; 生长期结束时 (2001 年 9 月底), 仔细数 每棵幼苗茎干上的芽数, 并采摘每棵树苗上所有的 树叶。将上述种子、树叶等以种子或幼苗为单位分 别装入纸袋中, 分生境按植株进行编号, 置烘箱于 $65{ }^{\circ} \mathrm{C}$ 条件下烘干至恒重 然后称重。

\section{4 数据分析}

实验设计是完全随机的, 方差分析用 SAS/STAT 软件中的 ANOVA 过程来实现( SAS 6.0)。在方差分 析中 SAS 模型以生境作为自变量, 以幼苗高生长速 度、子叶丢失、存活数量、每株幼苗的芽数、叶片重量 为因变量, 用邓肯多重比较和谢菲尔多重比较检验 在方差分析中有显著性差异的变量间的差异。

\section{3 结 果}

\section{1 坚果萌根与幼根伸长生长}

坚果播种 3 周后检查, 发现两种生境条件下坚 果留存率均为 $100 \%$, 萌根率林下为 $78.93 \% \pm$ $9.44 \%(n=400, p<0.001)$; 林缘为 $61.25 \% \pm$ $10.11 \%(n=400, p<0.001)$ 。次年夏初幼苗出土 前主根长度林下为 $(9.17 \pm 2.28) \mathrm{cm}(n=57, p<$ $0.05)$, 最长者达 $16.3 \mathrm{~cm}$, 最短仅 $5.4 \mathrm{~cm}$; 林缘为 $(16.09 \pm 2.80) \mathrm{cm}(n=63, p<0.05)$, 最长者达 23.2 $\mathrm{cm}$ 最短者达 $10.5 \mathrm{~cm}$ 。两种生境下坚果出土前侧 根均有所发育, 长度 5 15 mm 左右, 形成初步的根 系。

\section{2 幼苗子叶丢失}

土壤中留存辽东栎种子子叶丢失始于幼苗出土 前后，幼苗出土后 2 周时间内为丢失高峰期。辽东 栎幼苗期的子叶丢失有 3 种形式, 即:1) 仅丢失子 叶 幼苗其它部位未受伤害,2)整个幼苗连根被拖出 地面 3)在子叶与根颈的连接处被咬断主干。不同 生境子叶丢失的形式有较大的差异, 林缘样方的辽 东栎幼苗通常以仅丢失子叶或主干被咬断为主, 林 下样方则常整个幼苗被拖出地面, 而主干被咬断的 现象较少见(表 1)。

表 1 不同生境条件下辽东栎幼苗存活与死亡

Table 1 The survival and mortality of the seedlings of Quercus wutaishanica in different habitats

\begin{tabular}{|c|c|c|c|c|c|c|c|}
\hline \multirow{2}{*}{$\begin{array}{l}\text { 生境 } \\
\text { Habitats }\end{array}$} & \multicolumn{2}{|c|}{$\begin{array}{c}\text { 子叶丢失及相应死亡率 } \\
\text { Cotyledon loss and mortality }(\%)\end{array}$} & \multicolumn{4}{|c|}{$\begin{array}{c}\text { 死亡率 } \\
\text { Mortality }(\%)\end{array}$} & \multirow{2}{*}{$\begin{array}{c}\text { 成活率 } \\
\text { Survival rate } \\
(\%)\end{array}$} \\
\hline & $\begin{array}{l}\text { 丢失率 } \\
\text { Loss }\end{array}$ & $\begin{array}{c}\text { 死亡率 } \\
\text { Mortality }^{1)}\end{array}$ & $\begin{array}{c}\text { 仅子叶丢失 } \\
\text { Only cotyledon loss }\end{array}$ & $\begin{array}{c}\text { 主根被拖出 } \\
\text { Uprooted }\end{array}$ & $\begin{array}{l}\text { 主干被咬断 } \\
\text { Stem broken }\end{array}$ & $\begin{array}{c}\text { 干旱 } \\
\text { Drought }\end{array}$ & \\
\hline $\begin{array}{l}\text { 林下 } \\
\text { Under canopy }\end{array}$ & $11.74 \pm 7.71$ & $98.50 \pm 6.06$ & $11.44 \pm 7.34$ & $66.34 \pm 6.98$ & $8.41 \pm 5.09$ & $10.97 \pm 5.10$ & $3.11 \pm 5.02$ \\
\hline $\begin{array}{l}\text { 林缘 } \\
\text { Beside boundary }\end{array}$ & $29.69 \pm 6.28$ & $35.48 \pm 8.80$ & $10.60 \pm 3.77$ & $5.76 \pm 4.29$ & $50.88 \pm 3.93$ & $12.56 \pm 6.14$ & $19.89 \pm 4.91$ \\
\hline $\begin{array}{l}\text { 方差分析 } \\
\text { ANOVA }\end{array}$ & $F=65.29^{* * *}$ & $F=736.77^{* * *}$ & $F=0.21$ & $F=1093.83^{* * *}$ & $F=873.11^{* * *}$ & $F=0.79$ & $F=9040.74^{* * *}$ \\
\hline
\end{tabular}

1) The mortality of the acorns losing cotyledons only 
表 2 不同生境条件下幼苗高度、叶片数及芽数的比较

Table 2 Comparison of height, leaves and buds of Quercus wutaishanica seedlings in different habitats

\begin{tabular}{|c|c|c|c|c|c|}
\hline \multirow[b]{2}{*}{$\begin{array}{c}\text { 生境 } \\
\text { Habitat }\end{array}$} & \multicolumn{2}{|c|}{ 茎干 Shoot $($ mean \pm SD $)$} & \multicolumn{3}{|c|}{ 叶片 Leaves (mean $\pm \mathrm{SD})$} \\
\hline & $\begin{array}{c}\text { 高度 } \\
\text { Height }(\mathrm{cm})\end{array}$ & $\begin{array}{l}\text { 芽数 } \\
\text { Buds }\end{array}$ & $\begin{array}{c}\text { 数 } \\
\text { Number }\end{array}$ & $\begin{array}{c}\text { 长 } \\
\text { Length }(\mathrm{cm})\end{array}$ & $\begin{array}{c}\text { 干重 } \\
\text { Dry weight }(\mathrm{mg})\end{array}$ \\
\hline $\begin{array}{l}\text { 林下 } \\
\text { Under canopy }\end{array}$ & $4.74 \pm 0.84$ & $7.52 \pm 2.06$ & $3.56 \pm 0.79$ & $3.49 \pm 0.68$ & $42.27 \pm 17.62$ \\
\hline $\begin{array}{l}\text { 林缘 } \\
\text { Beside boundary }\end{array}$ & $7.94 \pm 1.30$ & $11.24 \pm 1.72$ & $5.62 \pm 1.51$ & $5.41 \pm 0.81$ & $81.14 \pm 30.97$ \\
\hline $\begin{array}{l}\text { 方差分析 } \\
\text { ANOVA }\end{array}$ & $F=16.86^{* * *}$ & $F=20.76^{* * *}$ & $F=18.04^{* * *}$ & $F=19.68^{* * *}$ & $F=11.18^{* * *}$ \\
\hline
\end{tabular}

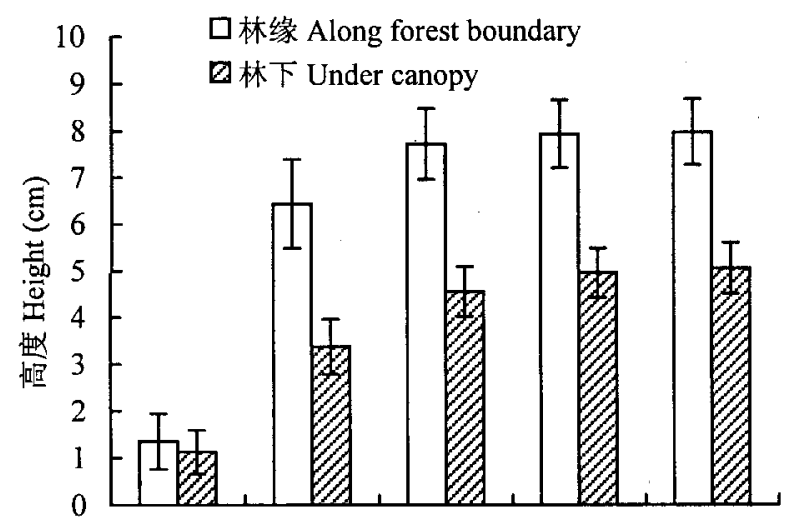

1st obs. 2nd obs. 3rd obs. 4th obs. 5th obs.

$(0 \mathrm{~d}) \quad(4 \mathrm{~d}) \quad(7 \mathrm{~d}) \quad(11 \mathrm{~d}) \quad(14 \mathrm{~d})$

观测时间 Observation time (d)

图 1 辽东栋幼苗出土后高生长速率

Fig.1 Height growth rate of Quercus wutaishanica seedlings after sprouting from soil

\section{3 辽东栎幼苗高生长}

林缘辽东栎幼苗出土较早且比较整齐,集中于 5 月底至 6 月初 林下较晚, 大部分幼苗在 6 月底至 7 月下旬陆续出土。一旦出土, 幼苗的茎干迅速进 行伸长生长,一般在 $2 \sim 3$ 周时间内达到最高点 (图 1) 然后在其顶部长出 1 5 片真叶, 通常 $3 \sim 4$ 片，几近轮生状排列。枝中下部的叶片不发育而呈 鳞片状，腋芽也很小。首次高生长结束后幼苗高度、 叶片和芽的数目等在不同生境条件下有所不同 (表 2)。位于林缘的幼苗 $50 \%$ 以上在首次高生长结束 后，其顶芽很快再次萌发进行第二次高生长，但生长 量很小, 长度仅有 $(1.31 \pm 0.69) \mathrm{cm}$, 叶片 $2 \sim 6$ 枚， 长 $(6.24 \pm 1.02) \mathrm{cm}(n=60)$,稍长于第一次生长的 叶片 $(5.43 \pm 1.74) \mathrm{cm}(n=60)$; 而林下幼苗则未发 现第二次生长现象。

\section{4 辽东栎幼苗的命运}

在本实验中, 播种后埋在土中的坚果基本得以 安全越过冬春季节 林下 20 个样方仅有 1 个样方丢
失 1 枚坚果, 林缘样方丢失率稍高于林下,20个样 方共丢失坚果 27 枚。进入翌年夏季, 随着露地凌晨 最低气温升至 $5{ }^{\circ} \mathrm{C}$ 以上, 白天 $20{ }^{\circ} \mathrm{C}$ 左右, 辽东栎坚 果开始萌芽出土。由于不同生境条件下气温的差 异 林下辽东栎幼苗的出土时间比林缘要晚 30 45 $\mathrm{d}$ 。当辽东栎幼苗出土时, 森林动物掘土捕食辽东栎 坚果的现象普遍发生, 它们拖拽幼苗的子叶使之在 子叶柄处与幼苗断裂分离, 或将整个幼苗连根拔出, 或在子叶着生处咬断幼苗的主干, 引起死亡(表 1)。

\section{4 讨 论}

\section{1 辽东栎种子秋季萌根及生长}

Watt(1919) 早已发现, 水分对保持栎类坚果的 活力十分重要, 当坚果失水到一定程度后即使再将 其置于水分充足的地方它也只是机械地吸水而不会 萌发。有人将坚果在地表萌发率低归结于坚果在地 表容易失水,从而导致种子失去活力 (Ovington \& MacRae , 1960 ; Kollmann \& Schill , 1996)。在我们的 试验中 林下播种的辽东係坚果萌发生根较早, 萌发 率较高, 说明林下土壤湿度条件较好, 能有效地维持 播种于林下的坚果水分平衡，促进坚果的萌发。而 林缘土壤, 尤其是表层土, 由于受较强的太阳光直 射、风吹等原因, 通常比较干燥, 这对于播种深度仅 $2 \mathrm{~cm}$ 左右的坚果来说, 无疑会影响坚果的水分平 衡, 从而对其萌发产生一定的影响, 导致了林缘坚果 萌发率较低。当坚果萌发之后, 为了维持肥大子叶 的水分平衡，适当的水分胁迫则可能又对主根的伸 长生长有促进作用。所以在翌年夏季幼苗出土前, 林缘坚果的主根长度显著大于林下, 为幼苗日后的 抗逆和生长发育奠定了较好的基础。

\section{2 幼苗期动物捕食、子叶丢失及后果}

据孟智斌等 (1997)、孙书存 (1998)、王巍 (2000) 等报道和我们的野外观察, 辽东栎种子的捕食动物 以啮齿类和鸟类为主, 前者有花鼠( Tamias 
sibiricus)、岩松鼠 (Sciurotamias davidianus)、大林姬鼠 (Apodemus speciosus)、社鼠 (Rattus confucianus)、大仓 鼠 (Cricetulus triton) 和棕背 $\square$ (Clethrionomys rufocanus) ,后者则多是松鸦 (Garrulus glandarius) 和褐马 鸡(Crossoptilon mantchuricum) 等。2000 年为辽东栎 结实丰年，可能是由于捕食者饱足 (Predator satiation) 的原因, 两种生境条件下覆土播种的辽东栎坚果都 有效地躲避了动物的捕食。第二年夏季辽东栎幼苗 出土前后,由于具有极强的嗅觉功能(孙书存等, 2001）, 以及春夏季节地面食物的缺乏，啮齿类开始 其活跃的地面活动来搜寻食物。当它们遇到辽东栎 幼苗时就会通过嗅觉来扒土临食埋藏在土壤中的辽 东栋种子子叶。表 1 显示, 子叶遭受捕食后对幼苗 的影响并非都是致命的，捕食后造成幼苗死亡的直 接原因是将子叶连同幼苗包括主根一起被拖出地 面。在枯枝落叶较多、土壤疏松的林下土壤中这种 现象经常发生, 而在林缘, 由于地表枯枝落叶和土壤 腐殖质含量均较少, 土壤质地较坚实, 同时由于林缘 幼苗在发芽出土前主根显著长于林下的幼苗, 使之 能够更牢固地与土壤结合在一起。当坚果受到捕食 动物攻击时, 由于受攻击的主要对象依然是坚果本 身, 即种子的肥大子叶, 所以往往被拖走的仅仅是子 叶, 其根系及胚芽或茎干通常会完好地保留在土中。 即使子叶丢失, 已经转移到主根的营养也足以维持 幼苗的生命和继续生长。另一种导致辽东栋幼苗死 亡的原因是, 由于幼苗子叶总是位于茎干的一侧, 如 果啮齿动物掘土时首先遇到幼苗的茎干, 出于本能 它们会首先咬断幼苗茎干，然后拖走位于茎干另一 侧的子叶。野外调查我们发现, 辽东栎幼苗的根部 不产生不定芽, 失去茎干的幼苗很难再萌芽生长, 从 而引起幼苗的死亡。

Sonesson(1994) 曾报道, 人为去除子叶对夏柇 ( Quercus robur) 幼苗的成活和生长的影响不显著。 显然，子叶丢失并不是导致幼苗建立失败的直接原 因。在我们的试验中, 丢失子叶幼苗的死亡率与未 丢失子叶幼苗因干旱而导致的死亡率十分接近, 丢 失子叶是否对幼苗生长量造成不良影响也不显著 (表 1)。这一结果暗示, 经过幼苗发芽出土前主根 的伸长生长, 辽东柇坚果已经把幼苗期所需的营养 转移到了根部, 从而及时地摆脱了幼苗成活和生长 对子叶的绝对依赖, 以提高生存率。而在林下, 由于 枯枝落叶含量较高, 土壤疏松 幼苗的子叶遭到捕食 时, 容易连根被拖出地面而引起死亡, 即使是仅丢失 子叶, 也由于林下幼苗主根较短, 从子叶转移来的营
养较少, 因而林下辽东栋幼苗对子叶营养储备的依 赖程度较高，所以丢失子叶后死亡率也较高 (表 1)。 另外, 林下土质较疏松, 如果主根未被拖出地面, 动 物拖走子叶的结果也会导致根系与土壤接触状态的 改变。在土壤水分匮乏时, 这种改变很容易造成幼 苗水分平衡失调, 从而引起幼苗死亡。这一结果也 许是对为什么林下常出现辽东栎幼苗密度小、建立 困难等现象 (孙书存, 1998; 王巍等, 2000) 的一种比 较合理的解释。

\section{3 幼苗补充、建立与生境条件}

辽东柇幼苗阶段表现出较强的喜光特性: 王巍 等(2000)发现, 林窗中辽东栋幼苗的高度、叶片面积 等方面都显著大于林冠下的同龄苗。通过对比试验 我们也发现, 油松人工林下的一年生辽东栎幼苗无 论是其高度、叶长、叶干重等都显著低于林缘的同龄 苗(表 2)，特别是林缘的辽东栋幼苗大多能够进行 第二次生长, 这种现象表明, 林缘较高的气温、较强 的光照、较长的光照时间和较大的昼夜温差等环境 条件, 有利于辽东柇幼苗在生长季节保持较高的生 长活力和较多的生物量积累, 为其生存和进一步生 长发育提供了物质基础, 有利于辽东柇幼苗的建立。 这一结果不仅与许多学者关于柇类的更新依赖干 扰 林下更新普遍不良，而林窗、林缘、采伐迹地等柇 类更新较好的结果一致 (Cho \& Boermer, 1991 ; Retana et al. 1992; Mayor \& Roda，1993; 孙书存,1998; 王巍，2000），而且较好地揭示了落叶栎类更新的生 态学特性。

\section{4 辽东栋林实生更新对策的探讨}

在栎林更新过程研究中, 人们发现位于地表的 柇类坚果很容易因水分的丧失而失去发芽能力, 另 一方面 柇树坚果与捕食动物之间存在着十分有趣 的现象，即栎树坚果的传播、萌发依赖于捕食动物的 活动，因为柇林下的环境条件不适应栎树幼苗的补 充与建立, 所以栎树坚果需要被捕食动物搬运并埋 藏起来，以维持其水分平衡，并得到尽快发芽生根的 机会, 而动物的搬运和埋藏的目的则是为其日后食 物短缺时取食 (Fox, 1982 ; Sonesson, 1994)。因此,了 解柇树是如何解决其实现既需要动物的搬运和埋 藏, 又不得不逃避被完全消费的危险之间的矛盾, 以 完成其更新与扩大分布区的使命，是探索柇林更新 对策的重要途径。

通常 落叶柇的坚果没有休眠期, 成熟脱落后只 要环境条件适宜, 其下胚轴立即萌发, 向下生长发育 成一个粗大的主根 (Fox ,1982), 使肥大子叶的水分 
平衡得以维持（Watt，1919；Ovington \& MacRae， 1960)。主根的萌发和根系的初步发育, 对于防止幼 苗被捕食者拖出地面而死亡起到了关键的作用，因 为此时捕食的结果往往仅是子叶的丢失。由于在幼 苗出土前子叶已将一部分营养转移到根系中, 当捕 食仅仅是丢失子叶时, 留在土中的主根和胚芽仍能 维持幼苗的生命和生长。因此, 我们认为对栎树来 说, 更重要的是坚果通过这种萌根方式将足够的营 养及早地转移到主根中, 避免发芽出土时整个种子 被捕食的厄运。由此可以推测, 栎树坚果胚根于当 年秋季落果后迅速萌发和快速生长, 是栎类树种的 种子适应捕食压力的一种有效对策。这种对策是柇 树与捕食者长期相互适应的结果: 捕食者将栎树坚 果搬运到林外比较适宜的地方埋藏起来，以作为日 后的食物来源; 由于被埋藏的栎树坚果更容易萌根 （Watt，1919 ;Jones，1959），这对坚果躲避其它捕食者 的捕食消耗、扩大栎林分布范围、提高栎树幼苗补充 和建立的有效性 (Ovington \& MacRae,1960; Sonesson , 1994 ;Kollmann \& Schill, 1996;王巍等，2000; 孙 书存等 ,2001; Zhang \& Wang ,2001)等方面，都有十分 重要的意义。由于栎类种子成熟脱落后很快萌发， 并将子叶中的营养迅速地向根部转移, 使幼苗的成 活和生长不再绝对地依赖其子叶的营养，从而逃避 了啮齿类捕食整个种子,危及幼苗生命的危险。上 述现象暗示, 在捕食动物对柇树种子的传播和消费 之间可能存在着另一种补偿平衡, 即栎树种子通过 及时萌发来转移一部分营养至新生的根部, 然后在 不影响或不显著影响幼苗的存活与生长的前提下， 将子叶提供给动物作为传播种子的 回报”。这种补 偿平衡无论对于柇树本身还是捕食者来说，显然都 是有利的。

\section{参 考 文 献}

Bacilieri, R., M. A. Bouchet, D. Bran, M. Grandjanny, M. Maistre, P. Perret \& F. Romane. 1993. Germination and regeneration mechanisms in Mediterranean degeneration forests. Journal of Vegetation Science, 4: $241 \sim 246$.

Bossema, I. 1979. Jays and oaks: an eco-ethological study of a symbiosis. Behaviours, 70: $1 \sim 117$.

Brookes, P.C., D. L. Wigston \& W.F. Bourne. 1980. The dependence of Quercus robur and Quercus petraea seedlings on cotyledon potassium, magnesium calcium and phosphorus during the first year of growth. Forestry, 53: $167 \sim 177$.

Chambers, J.C. \& J.A. Mackahon. 1994. A day in the life of a seed: movements and fates of seeds and their implications for natural and managed systems. Annual Review of Ecology and Systematics, 25: $263 \sim 293$.
Cho, D.S. \& R.E.J. Boermer. 1991. Canopies disturbance patterns and regeneration of Quercus species in two Ohio old-growth forest. Vegetatio, 93: 9 18.

Crawley, M.J. \& C.R. Long. 1995. Alternate bearing, predator satiation and seedling recruitment in Quercus robur L. Journal of Ecology, 83: $682 \sim 696$.

Ducrey, M. \& J. Toth. 1992. Effect of cleaning and thinning on height growth and girth increment in holm-oak coppices (Quercus ilex). Vegetatio, 99/100: $365 \sim 376$.

Espelta, J. M., M. Riba \& J. Retana. 1995. Patterns of seedling recruitment in west-mediterranean Quercus ilex forests influenced by canopies development. Journal of Vegetation Science, 6: 465 $\sim 472$.

Fox, J. F. 1982. Adaptation of gray squirrel behavior to autumn germination by white oak acorns. Evolution, 36: $800 \sim 809$.

Giovannini, G., D. Perulli, P. Piussi \& F. Salbitano. 1992. Ecology of vegetative regeneration after coppicing in macchia stands in central Italy. Vegetatio, 99/100: $331 \sim 343$.

Gao, X.M. (高贤明), W. Wang (王巍), X.J. Du (杜晓军) \& K.P. Ma (马克平) . 2001. Size structure, ecological significance and population origin of Quercus wutaishanica forest in Beijing Mountainous area. Acta Phytoecologica Sinica (植物生态学 报), 25:673 678. (in Chinese with English abstract)

Jarvis, P. G. 1963. The effects of acorn size and provenance on the growth of seedlings of sessile oak. Quarterly Journal of Forestry, 57: $11 \sim 19$.

Jones, E. W. 1959. Biological flora of the British Isles: Quercus L. Journal of Ecology, 47: 169 222 .

Kollmann, J. \& H.P. Schill. 1996. Spatial patterns of dispersal, seed predation and germination during colonization of abandoned grassland by Quercus petraea and Corylus avellana. Vegetatio, 125: $193 \sim 205$.

Mao, S.S. (茅世森) \& S. Song (宋凤山). 1997. The study on the climatic characteristics of the research site of Beijing Forest Ecosystem Research Station (BFERS). In: Chen, L.Z. (陈灵 芝) \& J.H. Huang (黄建辉) eds. The study on structure and function of forest in warm temperate zone. Beijing: Science Press. $28 \sim 37$. (in Chinese with English abstract)

Mayor, X. \& F. Roda. 1993. Growth response of holm-oak (Quercus ilex L.) to commercial thinning in the Montseny mountains (NE Spain). Annals of Forest Science, 50: $247 \sim 256$.

Meng, Z. B. (孟智斌) \& Z. B. Zhang (张知彬). 1997. The species and habitats of bird and mammal and the characteristics of rodent community in the mountain area of Beijing. In: Chen, L. Z. (陈灵芝) \& J. H. Huang (黄建辉) eds. The study on structure and function of forest ecosystem in warm temperate zone. Beijing: Science Press. 76 87. (in Chinese with English abstract)

Ovington, J.D. \& C. MacRae. 1960. The growth of seedlings of Quercus petraea. Journal of Ecology, 48: $549 \sim 555$.

Price, M. V. \& S. H. Jendins. 1986. Rodent as seeds consumers and dispersers. In: Murray, D.R. ed. Seed dispersal. Orlando: Academic Press. $191 \sim 235$.

Retana, J., M. Riba, C. Castell \& J. M. Espelta. 1992. Regeneration by sprouting of holm-oak (Quercus ilex) stands exploited by selection thinning. Vegetatio, 99/100: $355 \sim 364$.

Shaw, M. W. 1968a. Factors affecting the natural regeneration of 
sessile oak (Quercus petraea) in North Wales. 1. A preliminary study of acorn production, viability and losses. Journal of Ecology, 56: $565 \sim 583$.

Shaw, M. W. 1968b. Factors affecting the natural regeneration of sessile oak (Quercus petraea) in North Wales. 2. Acorn losses and germination under field condition. Journal of Ecology, 56: $647 \sim 660$.

Sonesson, L.K. 1994. Growth and survival after cotyledon removal in Quercus robur seedlings, grown in different national soil types. Oikos, 69: $65 \sim 70$.

Sun, S.C. (孙书存). 1998. Population biology of Quercus liaotungensis in Dongling Mountain region. Ph. D. dissertation of Institute of Botany, the Chinese Academy of Sciences. (in Chinese with English summary)

Sun, S.C. (孙书存) \& L.Z. Chen (陈灵芝). 2001. The effects of animal removal and groundcover on the fate of seeds of Quercus liaotungensis. Acta Ecologica Sinica (生态学报), 21:80 85. (in Chinese with English abstract)

Wang, W. (王巍) . 2000. Natural regeneration of Quercus liaotungensis Koidz. Ph. D. dissertation of Institute of Botany, the Chinese Academy of Sciences. (in Chinese with English summary)

Wang, W. (王巍), Q. K. Li (李庆康) \& K.P. Ma (马克平) . 2000. Establishment and spatial distribution of Quercus liaotungensis Koidz. seedlings in Dongling Mountain. Acta Phytoecologica Sinica(植物生态学报)，24：595 600. (in Chinese with English abstract)

Watt, A.S. 1919. On the causes of failure of natural regeneration in British oakwoods. Journal of Ecology, 7: 173 203.

Zhang, Z. B. \& F.S. Wang. 2001. Effect of burial on acorn survival and seedling recruitment of Liaodong oak (Quercus liaotungensis) under rodent predation. Acta Theriologica Sinica (兽类 学报), 21: $35 \sim 43$. 